\title{
Hyperuricemia and the risk for subclinical coronary atherosclerosis - data from a prospective observational cohort study
}

\author{
Eswar Krishnan ${ }^{*}$, Bhavik J Pandya², Lorinda Chung ${ }^{1}$ and Omar Dabbous ${ }^{2}$
}

\begin{abstract}
Introduction: Our purpose was to test the hypothesis that hyperuricemia is associated with coronary artery calcification (CAC) among a relatively healthy population, and that the extent of calcification is directly proportional to the serum uric acid ( $(\cup A)$ concentration.

Methods: Data from 2,498 participants in the Coronary Artery Risk Development in Young Adults (CARDIA) study were analyzed using logistic regression models. Subjects were free of clinical heart disease, diabetes, and renal impairment. The main measure was the presence of any CAC by computerized tomography (Agatston score $>0$ ).

Results: Forty-eight percent of the study participants were male and $45 \%$ were African-American. Mean ( \pm SD) age was $40 \pm 4$ years, body mass index $28 \pm 6 \mathrm{~kg} / \mathrm{m}^{2}$, Framingham risk score $-0.7 \pm 5 \%$, blood pressure $113 \pm 14 / 75 \pm$ $11 \mathrm{mmHg}$, alcohol consumption $12 \pm 27 \mathrm{ml} /$ day, and sUA $297 \pm 89 \mu \mathrm{mol} / \mathrm{L}(5.0 \pm 1.5 \mathrm{mg} / \mathrm{dL}$ ). Prevalence of CAC increased with sUA concentration among both men and women. Adjusted for age, gender, race, lipoproteins, triglycerides, smoking, blood pressure, presence of metabolic syndrome, C-reactive protein, waist circumference, alcohol use, creatinine, and serum albumin, the highest quartile of sUA (>393 $\mu \mathrm{mol} / \mathrm{L}[6.6 \mathrm{mg} / \mathrm{dL}]$ for men and $>274 \mu \mathrm{mol} / \mathrm{L}[4.6 \mathrm{mg} / \mathrm{dL}]$ for women) was associated with an odds ratio of 1.87 (1.19-2.93) compared to the lowest quartile (<291 $\mu \mathrm{mol} / \mathrm{L}[4.9 \mathrm{mg} / \mathrm{dL}]$ for men and $<196 \mu \mathrm{mol} / \mathrm{L}[3.3 \mathrm{mg} / \mathrm{dL}]$ for women). Among those with any CAC, each unit increase in $S \cup A$ was associated with a $22 \%$ increase in Agatston score $(P=0.008)$ after adjusting for the above covariates.
\end{abstract}

Conclusions: Hyperuricemia is an independent risk factor for subclinical atherosclerosis in young adults.

\section{Introduction}

Although the link between elevated serum uric acid (sUA) concentrations and the risk for atherosclerotic cardiovascular and cerebrovascular disease has long been observed, only recently have the pathophysiologic links become clearer [1]. Kanbay and colleagues [2] recently summarized the emerging data suggesting that hyperuricemia may cause not only atherosclerosis in the macrovascular beds such as the coronaries and the carotids but also microvascular damage in the renal vascular bed and may exacerbate vascular disease.

Almost all epidemiological studies performed in populations of higher-than-normal risk have shown a consistent

\footnotetext{
* Correspondence: e.krishnan@stanford.edu

'Department of Medicine, Stanford University School of Medicine, 1000

Welch Road, Suite 203, Palo Alto, CA 94304, USA Full list of author information is available at the end of the article
}

association between sUA and coronary artery disease (CAD) [1]. Studies on the lower-than-normal-risk populations that have relatively few events need to have a very large sample size to be able to measure the magnitude of relative risks observed in the high-risk groups (relative risk of 1.5 to 2.5). In such a context, markers of subclinical atherosclerosis are important outcomes to examine. The detection of coronary artery calcification (CAC) by ultrafast computed tomography $(\mathrm{CT})$ scanning is highly predictive of the presence of histopathologic atherosclerosis [3], and the extent of calcification correlates well with plaque burden [4]. It is also an accurate (positive predictive value of $84 \%$ to $96 \%$ ) measure of obstructive CAD compared with angiographic evaluation and is a useful tool to study subclinical CAD, especially in population settings [5]. Some argue that, in the setting of observational studies, CAC measurement may even be superior to other measures
C Biomed Central 
such as carotid intima-media thickness in predicting cardiovascular outcomes [6].

The primary objective of this epidemiological study was to understand the relationship between sUA concentration and CAC in relatively young and healthy adults. If the hyperuricemia-CAD link is real, we can expect that the prevalence of CAC among those with higher sUA levels will be greater and that the extent of CAC will be directly proportional to the degree of hyperuricemia - a hypothesis that we tested here.

\section{Materials and methods Design}

We performed cross-sectional analyses of year-15 data from the Coronary Artery Risk Development in Young Adults (CARDIA) study, a prospective observational cohort study of 5,115 subjects recruited between the ages of 18 and 30 years and followed for 15 years. Ethical approval for the CARDIA study was obtained from participating institutions, and informed consent was obtained from the patients.

\section{Setting, participants, and follow-up}

The CARDIA study is an ongoing multicenter cohort study based at four centers: Chicago, IL; Birmingham, AL; Minneapolis, MN; and Oakland, CA. The observation baseline of this study was 1985-1986, when all participants were recruited and enrolled. The cohort had approximately equal numbers of African-Americans and whites, men and women, adults 18 to 24 years old and 25 to 30 years old, and participants with more than and less than high school education [7]. Subsequently, they were followed up at years 2, 5, 7, 10, 15, and 20. A detailed description of the study methodology has been published [7]. At baseline and every follow-up visit, CARDIA study participants underwent extensive medical examinations with a specific focus on cardiovascular risk factors. The study provided detailed information on demographic characteristics and on lifestyle habits such as alcohol consumption and smoking.

\section{Inclusion and exclusion criteria}

We studied the data collected during year 15 of this prospective study, at which time all participants were invited to obtain an electron beam computerized tomography (EBCT) scan. We excluded all participants with missing values for CAC scores or sUA concentrations and any self-reported coronary heart disease, including angina symptoms. Since diabetes is associated with both higher sUA concentration and higher incidence rates of CAC [8], we excluded all subjects with type 2 diabetes or prediabetes (defined by American Diabetes Association criteria [9]) and those who reported the use of diabetes medications or a physician diagnosis of diabetes. Since the presence of renal impairment can affect sUA concentration and atherosclerosis, individuals with an estimated glomerular filtration rate of less than $60 \mathrm{~mL} /$ minute per $1.73 \mathrm{~m}^{2}$ (calculated by the Modification of Diet in Renal Disease equation) were also excluded [10].

\section{Coronary artery calcification measurement, case definition, and rationale}

In a single session, two CT scans were obtained at year 15 for each participant by using an EBCT scanner (Imatron C-150 ${ }^{\mathrm{TM}}$; GE Medical Systems, Milwaukee, WI [Chicago and Oakland centers]) or a multidetector CT scanner (GE Lightspeed $^{\mathrm{TM}}$; GE Medical Systems [Birmingham center] or Volume Zoom ${ }^{\mathrm{TM}}$; Siemens, Erlangen, Germany [Minneapolis center]). Details of the CT protocol have been published [11]. The amount of CAC can be measured to provide a reasonable estimate of total coronary atheroma, including calcified and non-calcified plaque. Coronary calcium assessments for diagnosis of atherosclerosis and obstructive disease and for risk stratification for future cardiac events have undergone significant validation over the past 20 years $[12,13]$. The extent of calcification was quantified by using the Agatston method, in which total calcium scores were calculated on the basis of the number, areas, and peak Hounsfield computed tomographic numbers of the calcific lesions [4]. A previous study showed that an Agatston score of zero indicates no identifiable plaque with a negative predictive value of $98 \%$ for those 40 to 49 years old, an age group similar to that of our cohort [4]. In angiographic studies done in older populations, scores of 1 to 99 indicate mild plaque, 100 to 399 moderate plaque, and at least 400 severe atherosclerotic plaque burden. Given that our goal was to assess for any CAC in young adults with no clinical evidence of $\mathrm{CAD}$, we defined $\mathrm{CAC}$ as any positive, non-zero Agatston score, using the average of two scans. Each scan with at least one non-zero score $(n=350,11.5 \%)$ was reviewed by an expert investigator who was blinded to the scan scores to verify CAC presence. The agreement between scans was high (kappa $=0.79,95 \%$ confidence interval $[\mathrm{CI}] 0.75$ to 0.83 ), and discordance was only $3.6 \%$ [14].

\section{Serum uric acid}

Fasting concentration of sUA was measured in a central laboratory by using a colorimetric assay with rigorous quality control.

\section{Statistical analyses}

Our primary outcome measure was subclinical atherosclerosis, defined as the presence of CAC by CT scan. Several cutoff points ranging from 0 to 1,000 have been used in other studies to define the presence of CAC, depending on its prevalence. We assessed the CT images for evidence of any CAC (defined as Agatston 
score of greater than 0 ) and for the presence of at least mild plaque (Agatston score of greater than 10). The choice of these cutoffs was dictated by the statistical distribution of Agatston score in our young population.

The first objective was to examine the relationship between measures of subclinical atherosclerosis and sUA (as a continuous as well as a stratified measure). These analyses were performed by using logistic regression models in which presence or absence of CAC (defined as an Agatston score of greater than 0 or greater than 10) was the dependent variable and sUA was the independent variable of interest. sUA quartiles were defined for men and women separately and were subsequently pooled.

We adjusted for the following covariates measured at year 15: age, gender, race, high- and low-density lipoproteins, triglycerides, smoking, blood pressure stage [15], presence of metabolic syndrome [16], C-reactive protein, waist circumference, alcohol use, creatinine, and serum albumin concentration. These factors have been assessed in previous studies of hyperuricemia and cardiovascular risk and therefore were included in the present analyses. In our primary analyses, all of these covariates were included in the model, regardless of the statistical significance of each. Subsequent confirmatory analyses deployed backward selection methods (with $P<$ 0.20 as the cutoff) to derive a more parsimonious model.

The second objective of the analyses was to test the hypothesis that, among those with CAC, Agatston scores will be directly proportional to sUA concentration. The distribution of these scores was skewed, with numerous outliers. Hence, in these analyses, we used ordinary least square (OLS) regression models in which the dependent variable was the log-transformed Agatston score $\left(\log _{2}[C A C+1]\right)$. For these OLS regression analyses, we excluded all participants who had an Agatston score of zero.

In all regression models, we explored the data for the presence of statistical interaction between gender, race, and sUA. Model fit was verified by using the HosmerLemeshow method [17]. Data analyses were performed by using SAS $^{\circledR}$ (SAS Institute Inc., Cary, NC, USA).

\section{Results}

Of the 5,115 participants at baseline, 3,671 participated in the examination at year 15 . Among these, 1,173 were excluded as they met the study exclusion criteria. Overall, there were 2,498 participants $(1,211$ men and 1,287 women) available for analyses. Table 1 shows the characteristics of these participants. Higher sUA concentrations were associated with greater prevalence of cardiovascular risk factors, metabolic syndrome, and high Framingham risk score (Table 1). Fewer than 20 participants had a self-reported history of probable or definite gout and these could not be independently verified. None of the study population was using urate-lowering medications such as allopurinol, probenecid, sulfinpyrazone, or losartan.

The mean \pm standard deviation of sUA concentration was $345 \pm 77 \mu \mathrm{mol} / \mathrm{L}(5.8 \pm 1.3 \mathrm{mg} / \mathrm{dL})$ for men and $238 \pm$ $65 \mu \mathrm{mol} / \mathrm{L}(4.0 \pm 1.1 \mathrm{mg} / \mathrm{dL})$ for women $(P<0.001)$. sUA was distributed normally among men and women, whites, and African-Americans. The proportions of participants with sUA of greater than $416 \mu \mathrm{mol} / \mathrm{L}(7.0 \mathrm{mg} / \mathrm{dL})$ were 8.4\% $(n=211)$ overall, $16.6 \%(n=201)$ among men, and $0.8 \%(n=10)$ among women. The men had a significantly worse overall cardiovascular risk profile than women (Table 1). sUA concentrations were correlated with male gender (correlation coefficient of 0.6; $P<0.01$ ) and Framingham risk score $(0.52 ; P<0.01)$ but were only modestly associated with other known cardiovascular risk factors (all correlation coefficients of less than $0.3 ; P<0.05)$. C-reactive protein levels were not correlated with sUA $(P=0.20)$.

The majority $(90.5 \%, n=2,260)$ of participants had no detectable CAC. Overall, 9.5\% $(n=238)$ of participants had an Agatston score of greater than 0, 6.3\% $(n=158)$ had an Agatston score of greater than 10, and 1.4\% $(n=$ 34) had an Agatston score of greater than 100. As expected in a cohort free of clinical CAD, relatively few participants had an Agatston score of greater than 400 $(n=4)$. Among those with any CAC, mean scores were higher in men than in women (75.0 versus 60.8 ; Wilcoxon rank sum test $P=0.77$ ) and in whites than in African-Americans (71.6 versus 70.0; $P=0.19$ ), but these findings were not statistically significant.

At each quartile of sUA, men had a greater prevalence of CAC than women (Figure 1). However, the prevalence of CAC increased with increasing sUA in both genders, and the highest quartile had almost two times the prevalence compared with the lowest (odds ratio [OR] 1.87, CI 1.19 to 2.93) (Table 2).

In the bivariate logistic regressions in which presence or absence of CAC was the dependent variable, the highest quartile of sUA concentrations (greater than 393 $\mu \mathrm{mol} / \mathrm{L}[6.6 \mathrm{mg} / \mathrm{dL}]$ for men and greater than 274 $\mu \mathrm{mol} / \mathrm{L}[4.6 \mathrm{mg} / \mathrm{dL}]$ for women) had an OR of greater than 2.0 among both men and women, regardless of the Agatston score cutoff used to define CAC (Table 3).

We developed parallel multivariable logistic regression models that included all of the risk factors of interest (age, gender, race, high- and low-density lipoproteins, triglycerides, smoking, blood pressure class, presence of metabolic syndrome, C-reactive protein, waist circumference, alcohol use, creatinine, and serum albumin concentration) in the model. Data were pooled for men and women, and gender-specific stratification for quartiles of sUA was used after we established that there was no statistically significant interaction between gender and 
Table 1 Characteristics of study population by gender and serum uric acid quartile

\begin{tabular}{|c|c|c|c|c|c|c|c|c|}
\hline & \multicolumn{4}{|c|}{ Women $(n=1,287)$} & \multicolumn{4}{|c|}{ Men $(n=1,211)$} \\
\hline & Quartile 1 & Quartile 2 & Quartile 3 & Quartile 4 & Quartile 1 & Quartile 2 & Quartile 3 & Quartile 4 \\
\hline sUA range, $\mu \mathrm{mol} / \mathrm{L}[\mathrm{mg} / \mathrm{dL}]$ & 77-196 [1.3-3.3] & $196-226[3.3-3.8]$ & $232-274[3.9-4.6]$ & $280-636[4.7-10.7]$ & $155-291[2.6-4.9]$ & $297-333[5.0-5.6]$ & $339-393[5.7-6.6]$ & $399-690[6.7-11.6]$ \\
\hline Age, years & $40.1(3.8)$ & 40.1 (3.6) & $40.3(3.6)$ & $40.5(3.8)$ & $40.1(3.6)$ & $39.8(3.5)$ & $40.4(3.5)$ & $40.4(3.6)$ \\
\hline African-American & $43.5 \%$ & $42.6 \%$ & $46.5 \%$ & $57.5 \%$ & $42.7 \%$ & $37.4 \%$ & $42.5 \%$ & $43.2 \%$ \\
\hline Body mass index, $\mathrm{kg} / \mathrm{m}^{2}$ & $24.8(4.7)$ & $27.2(6.2)$ & $29.6(6.8)$ & $33.1(7.2)$ & $26.1(3.8)$ & $27.4(4)$ & $28.1(4.5)$ & $30.1(4.6)$ \\
\hline Alcohol, mL/day & $6.2(10)$ & $5.7(11.8)$ & $9(32.7)$ & $9.1(22)$ & $13.3(35.4)$ & $14.2(25.1)$ & $16.2(24.4)$ & $20.3(35.1)$ \\
\hline Smokers & $36.6 \%$ & $39.7 \%$ & $38 \%$ & $46.2 \%$ & $35.3 \%$ & $39.4 \%$ & $39.3 \%$ & $40.2 \%$ \\
\hline Systolic BP, mm Hg & $107(12.5)$ & $109.3(14)$ & $112.4(15.3)$ & $115.5(16.3)$ & $111.7(11.2)$ & $114.8(13.5)$ & $115.4(12.9)$ & $118.3(14.7)$ \\
\hline Diastolic BP, mm Hg & $69.6(10.1)$ & $71.5(10.2)$ & $73.3(10.9)$ & 75.9 (12.6) & $73.3(9.2)$ & $76.3(11)$ & $76.8(10.2)$ & $79.2(12.1)$ \\
\hline $\begin{array}{l}\text { Serum fasting glucose, } \mathrm{mmol} / \mathrm{L} \\
{[\mathrm{mg} / \mathrm{dL}]}\end{array}$ & $\begin{array}{c}4.45(0.40)[80.2 \\
(7.2)]\end{array}$ & $\begin{array}{c}4.48(0.43)[80.7 \\
(7.7)]\end{array}$ & $\begin{array}{c}4.5(0.45)[81.1 \\
(8.1)]\end{array}$ & $\begin{array}{c}4.7(0.53)[84.3 \\
(9.5)]\end{array}$ & $4.7(0.46)[84(8.3)]$ & $\begin{array}{c}4.8(0.53)[85.7 \\
(9.6)]\end{array}$ & $\begin{array}{c}4.8(0.52)[86.4 \\
(9.4)]\end{array}$ & $4.9(0.54)[88.8(9.7)]$ \\
\hline Serum HDL-C, mmol/L [mg/dL] & $\begin{array}{c}1.54(0.33)[59.7 \\
(12.8)]\end{array}$ & $\begin{array}{c}1.44(0.35)[55.5 \\
\quad(13.5)]\end{array}$ & $\begin{array}{c}1.45(0.36)[56 \\
(14.1)]\end{array}$ & $\begin{array}{c}1.32(0.37)[51 \\
(14.4)]\end{array}$ & $\begin{array}{c}1.24(0.33)[48 \\
(12.9)]\end{array}$ & $\begin{array}{c}1.19(0.32)[45.9 \\
(12.4)]\end{array}$ & $\begin{array}{c}1.14(0.31)[43.9 \\
(11.8)]\end{array}$ & $\begin{array}{c}1.10(0.33)[42.4 \\
(12.6)]\end{array}$ \\
\hline Serum LDL-C, mmol/L [mg/dL] & $\begin{array}{c}2.72(0.73)[105.1 \\
(28.2)]\end{array}$ & $\begin{array}{c}2.74(0.74)[105.9 \\
(28.7)]\end{array}$ & $\begin{array}{c}2.80(7.7)[108.4 \\
(29.8)]\end{array}$ & $\begin{array}{c}3.01(0.79)[116.3 \\
(30.6)]\end{array}$ & $\begin{array}{c}2.87(0.74)[110.9 \\
(28.5)]\end{array}$ & $\begin{array}{c}3.12(0.81)[120.5 \\
(31.5)]\end{array}$ & $\begin{array}{c}3.19(0.95)[123.5 \\
(36.6)]\end{array}$ & $\begin{array}{l}3.23(0.92)[125 \\
(35.7)]\end{array}$ \\
\hline $\begin{array}{l}\text { Serum triglycerides, } \mathrm{mmol} / \mathrm{L} \\
{[\mathrm{mg} / \mathrm{dL}]}\end{array}$ & $\begin{array}{c}0.78(0.38)[68.8 \\
(33.8)]\end{array}$ & $\begin{array}{l}0.89(0.44)[78.7 \\
(39.1)]\end{array}$ & $\begin{array}{l}0.95(0.52)[84.5 \\
(45.8)]\end{array}$ & $\begin{array}{c}1.27(0.80)[112.6 \\
(70.7)]\end{array}$ & $\begin{array}{l}1.01(0.67)[89.8 \\
(59.7)]\end{array}$ & $\begin{array}{c}1.26(0.76)[111.5 \\
(67.4)]\end{array}$ & $\begin{array}{c}1.52(1.87)[135 \\
(165.7)]\end{array}$ & $\begin{array}{c}1.86(1.76)[164.8 \\
(156.1)]\end{array}$ \\
\hline $\begin{array}{l}\text { Serum creatinine, } \mu \mathrm{mol} / \mathrm{L}[\mathrm{mg} / \\
\mathrm{dL}]\end{array}$ & $79.6(8.8)[0.9(0.1)]$ & $79.6(8.8)[0.9(0.1)]$ & $\begin{array}{c}79.6(8.8)[0.9 \\
(0.1)]\end{array}$ & $79.6(8.8)[0.9(0.1)]$ & $97.2(8.8)[1.1(0.1)]$ & $97.2(8.8)[1.1(0.1)]$ & $\begin{array}{c}97.2(17.7)[1.1 \\
(0.2)]\end{array}$ & $97.2(17.7)[1.1(0.2)]$ \\
\hline Waist circumference, $\mathrm{cm}$ & $76.6(10.1)$ & $81.6(12.1)$ & $86.3(13.1)$ & $94(14.1)$ & $87.8(9.5)$ & $91.8(9.6)$ & $93.5(11)$ & $98.4(10.9)$ \\
\hline eGFR, abbreviated MDRD & $87.2(15.1)$ & $85.6(15.5)$ & $83.9(14.2)$ & $82.7(15.2)$ & 89.9 (13.6) & $88.3(14.1)$ & $87.1(19.3)$ & $85.6(16.1)$ \\
\hline Framingham risk score & $-4.8(4.1)$ & $-3.7(4.3)$ & $-3.3(4.4)$ & $-1.4(4.3)$ & $1.1(2.2)$ & $1.8(2.4)$ & $2.3(2.3)$ & $2.7(2.4)$ \\
\hline C-reactive protein, mg/dL & $1.5(2.9)$ & $1.5(1.7)$ & $1.6(2.9)$ & $1.8(1.7)$ & $1.7(2.9)$ & $1.8(2.0)$ & $2.0(2.5)$ & $2.1(2.5)$ \\
\hline
\end{tabular}

Data are presented as range, mean (standard deviation), or percentage. BP, blood pressure; eGFR, estimated glomerular filtration rate; HDL-C, high-density lipoprotein cholesterol; LDL-C, low-density lipoprotein cholesterol; MDRD, modification of diet in renal disease; sUA, serum uric acid. 


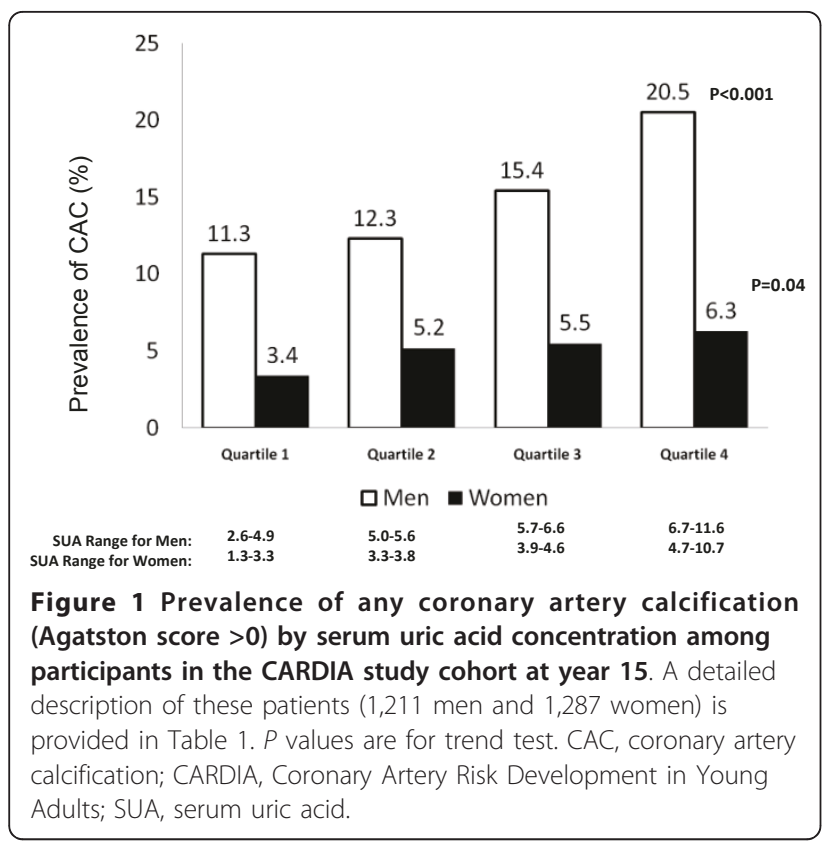

the correlation between sUA concentration and CAC (Table 2). Although these models differed in the definition of subclinical atherosclerosis and in the stratification strategy for sUA, all models showed that the highest quartile of sUA concentration was associated with significantly higher risk for subclinical atherosclerosis. In both multivariable regression models, each unit increase in sUA concentration was associated with an OR of 1.23 (CI 1.09 to 1.39) for subclinical atherosclerosis. The findings were replicated in backward stepwise selection models that eliminated those factors that were not significant individually as well as those in which an alternate stratification strategy for sUA was used (data not shown).

The last set of analyses focused on the association between sUA concentration and the severity of CAC. These analyses included only those subjects who had Agatston score of greater than zero $(n=238)$. Although the Agatston scores were higher among those with higher sUA concentrations (Figure 2), bivariate correlation analyses showed that the association was not strong (correlation coefficient of 0.13). However, in multivariable OLS regression models, each unit increase in sUA concentration was associated with a significant increase in the logtransformed Agatston score (beta coefficient of 0.288, 95\% CI 0.078 to $\left.0.498 ; P=0.008 ; \mathrm{R}^{2}=0.197 \%\right)$. In other words, there was an approximately $22 \%$ increase in Agatston score for each unit increase in sUA. When examined separately for each gender, this association persisted for men (beta coefficient of 0.300 , CI 0.078 to 0.522 ) and women (beta coefficient of $0.318, \mathrm{CI}-0.502$ to 1.138 ) but was statistically significant only in the former $(P=0.009)$.

\section{Discussion}

The association between hyperuricemia and the presence of subclinical atherosclerosis has not previously been studied in a cohort of young adults with no risk factors for CAD. Our study found a direct correlation between the prevalence and severity of CAC and sUA concentration in both men and women. This supports the hypothesis that uric acid may be involved in the pathologic process of atherosclerosis independently of conventional risk factors.

Table 2 Crude risk of increasing serum concentrations of uric acid

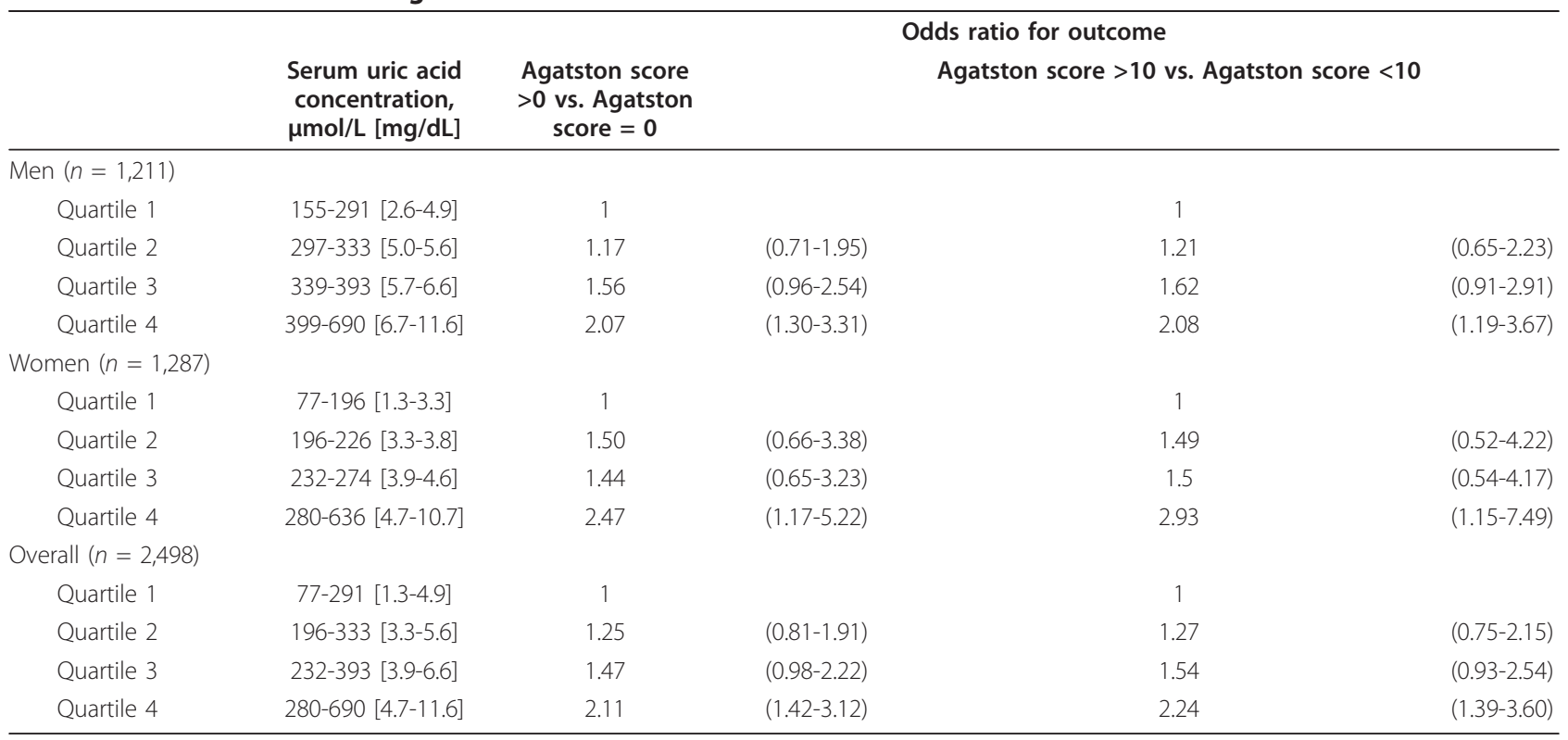




\begin{tabular}{|c|c|c|c|}
\hline & \multirow[b]{2}{*}{$\begin{array}{l}\text { Serum uric acid concentration, } \mu \mathrm{mol} / \mathrm{L} \\
{[\mathrm{mg} / \mathrm{dL}]}\end{array}$} & \multicolumn{2}{|c|}{ Odds ratio for outcome } \\
\hline & & $\begin{array}{c}\text { Agatston score }>0 \text { vs. Agatston } \\
\text { score }=0\end{array}$ & $\begin{array}{c}\text { Agatston score }>10 \text { vs. Agatston } \\
\text { score }<10\end{array}$ \\
\hline \multicolumn{4}{|c|}{ Quartile of serum uric acid ${ }^{a}$} \\
\hline Quartile 1 & $77-291[1.3-4.9]$ & 1 & 1 \\
\hline Quartile 2 & $196-333[3.3-5.6]$ & $1.24(0.78-1.97)$ & $1.26(0.72-2.22)$ \\
\hline Quartile 3 & 232-393 [3.9-6.6] & $1.42(0.9-2.24)$ & $1.50(0.87-2.58)$ \\
\hline Quartile 4 & $280-690[4.7-11.6]$ & $1.87(1.19-2.93)$ & $1.91(1.12-3.26)$ \\
\hline
\end{tabular}

Adjusted for the effects of age, gender, race, high- and low-density lipoproteins, triglycerides, smoking, blood pressure class, presence of metabolic syndrome, C-reactive protein, waist circumference, alcohol use, creatinine, and serum albumin concentration. No participants had diabetes or renal impairment. ${ }^{\mathrm{a}}$ Men and women were classified into quartiles by gender-specific cutoff numbers and were subsequently pooled.

Uric acid is a ubiquitous antioxidant in the blood [18]. Abnormally high serum concentrations of uric acid indicate oxidative stress, endothelial dysfunction, and slow coronary artery blood flow $[19,20]$. Elevated sUA concentration signifies a milieu with high oxidative stress and potentially indicates a vascular pathologic process such as atherosclerosis [21]. An association between hyperuricemia and CAC has been observed in previous studies involving patients with underlying risk factors for CAD such as type 1 diabetes, longstanding hypertension, or metabolic syndrome [22-25]. Importantly, a cross-sectional analysis of 443 individuals with type 1 diabetes suggested that the chances of progressive CAC were proportional to the magnitude of sUA concentration [22]. Another study involved older age groups compared with our cohort but also found that the correlation between uric acid and CAC was evident among men and women and in similar magnitude [13]. In the INSIGHT (International Nifedipine Study Intervention as Goal for Hypertension Therapy) study, in which CAC was measured in hypertensive patients who were older

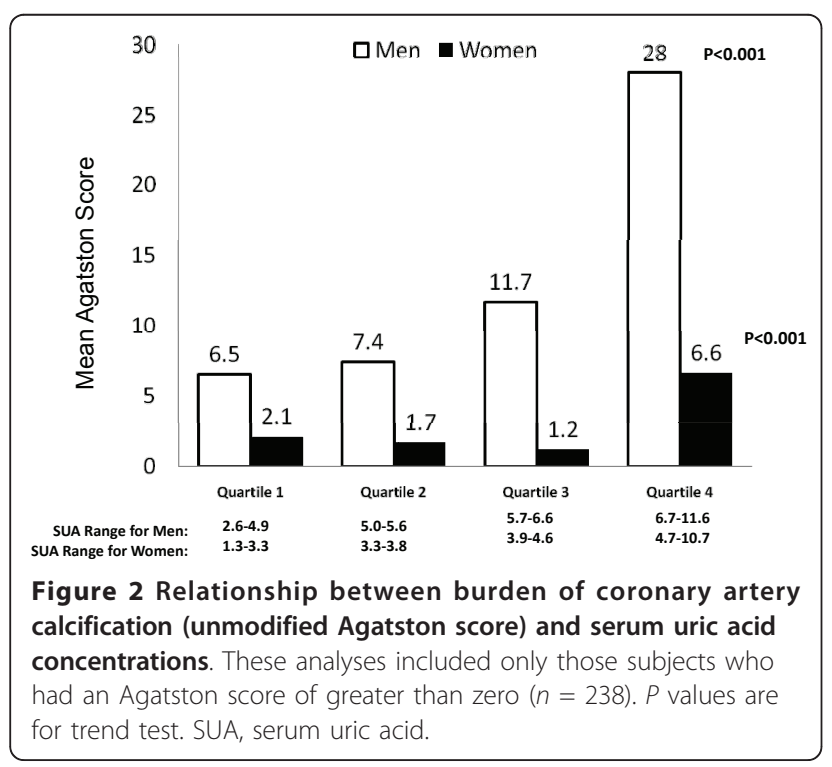

than 55 years of age and who had at least one more major cardiovascular risk factor, those with a total coronary calcium score (TCS) of greater than zero had a slightly higher sUA concentration compared with those with a TCS of zero $(333 \pm 89$ versus $315 \pm 83 \mu \mathrm{mol} / \mathrm{L}$ [5.6 \pm 1.5 versus $5.3 \pm 1.4 \mathrm{mg} / \mathrm{dL}$ ]; $P=0.03$ ) [23]. However, other studies, including the GENOA (Genetic Epidemiology Network of Arteriopathy) study on sibships with at least two members with diagnosed hypertension, did not show an association between uric acid concentration and the presence or severity of CAC after adjustment for conventional risk factors [25-27]. In addition, the National Heart, Lung and Blood Institute (NHLBI) Family Heart Study did not find a significant relationship between hyperuricemia and CAC in either gender [28]. Among those who underwent coronary angiography for suspected CAD, sUA concentration of greater than $416 \mu \mathrm{mol} / \mathrm{L}(7.0 \mathrm{mg} / \mathrm{dL})$ was associated with stable plaques without evidence of remodeling. The authors interpret this as suggesting that uric acid is a marker of atherosclerosis rather than a pathogenic mediator $[13,29]$.

Coronary atherosclerosis is less likely to be associated with calcification among women compared with coronary atherosclerosis with a similar degree of lumen narrowing in men [30]. In the CARDIA study cohort, the prevalences of CAC were approximately $15 \%$ among men and approximately 5\% among women overall [14]. This could be because younger women may be 'resistant' to atheroma growth [31].

Gender might be an important effect modifier in the association between hyperuricemia and CAC because of differences in (a) the distribution of sUA and (b) the prevalence of CAC. Iribarren and colleagues [32] analyzed data from the Atherosclerosis Risk in Communities (ARIC) study and concluded that an association between sUA and cardiovascular risk is evident in men but not women. In contrast, a similar study by Ishizaka and colleagues [33] reported that gender was not a factor. Since the prevalences of hyperuricemia and CAC are both lower among women, a greater sample size 
would be needed to detect a given effect size of hyperuricemia-CAC association. We defined quartiles separately for men and women prior to pooling. Statistical tests of gender-sUA interaction were not significant in our data. In gender-specific analyses, the direction and magnitude of risk among women were similar to those among men; however, the standard errors were wide because of the lower power for precise estimates.

The major strength of our community-based study is the generalizability of our results to young adults, including men, women, African-Americans, and whites. All of the studies described earlier were performed among patients with greater-than-normal cardiovascular risk, such as those with hypertension, diabetes, metabolic syndrome, psoriasis, or renal disease [13,21,23,26-29].

The primary limitation of this study was the cross-sectional nature of data analysis. Survivor bias can affect cross-sectional data analyses in that those with more severe disease die prior to the time point of analysis; however, this is not a major consideration in the CARDIA study as the main cause of mortality in the first 16 years was non-cardiovascular in the vast majority of patients (117/127 deaths out of 5,115 enrollees). Our future studies will examine the rate of progression of CAC over time among patients with hyperuricemia or gout or both. Gouty arthritis has been associated with CAD among middle-aged men [34], but our study had too few participants with gout $(\mathrm{n}<20)$ to allow a formal analysis. A larger number of women would have enabled separate analyses with respect to use of exogenous hormones and menstrual status. Owing to the design of our study, there was relatively little heterogeneity with respect to age (approximately 12 years), precluding an analysis of impact of age on the hyperuricemia-CAC association. However, in our analyses, age was not significantly associated with sUA concentration. sUA concentration is known to vary with time of day and recent dietary intake and possibly with physical exertion, introducing 'random noise' in sUA data. Unless sUA increases due to such variables can be shown to occur preferentially among those with higher CAC scores, this issue cannot explain our findings. As in all other epidemiological studies, unmeasured covariates could have caused residual confounding in our study as well.

\section{Conclusions}

We have shown for the first time that sUA is associated with the presence and severity of CAC in young healthy adults, implicating a potential role of uric acid in the pathogenesis of subclinical atherosclerosis. Our data are consistent with the growing body of literature that implicates the vascular injury associated with hyperuricemia - both macrovascular and microvascular $[2,22,35]$.

\section{Abbreviations}

CAC: coronary artery calcification; CAD: coronary artery disease; CARDIA: Coronary Artery Risk Development in Young Adults; Cl: confidence interval; $C T$ : computed tomography; EBCT: electron beam computerized tomography; OLS: ordinary least square; OR: odds ratio; sUA: serum uric acid; TCS: total coronary calcium score.

\section{Acknowledgements}

We appreciate the assistance of Sean Coady, of the National Heart Lung and Blood Institute (NHLBI), for obtaining data sets and providing helpful comments. The CARDIA study is conducted and supported by the NHLBI in collaboration with the CARDIA Study Investigators. This article was prepared using a limited access data set that EK obtained from the NHLBI and does not necessarily reflect the opinions or views of the CARDIA study or the NHLBI. Editing and bibliography assistance provided by Manel Valdes-Cruz, of Takeda Pharmaceuticals North America, Inc., is gratefully acknowledged.

\section{Author details}

${ }^{1}$ Department of Medicine, Stanford University School of Medicine, 1000 Welch Road, Suite 203, Palo Alto, CA 94304, USA. ²Department of Global Health Economics and Outcomes Research, Takeda Pharmaceuticals International, Inc., One Takeda Parkway, Deerfield, IL 60015, USA.

\section{Authors' contributions}

EK conceived of the manuscript idea, designed the analysis plan, performed statistical analysis, interpreted the results, and wrote the first draft of the manuscript with assistance from all other authors. He has possession of raw data sets and takes responsibility for the integrity of the data and the accuracy of the data analysis. Takeda Pharmaceuticals International, Inc. did not have access to the raw data, and Takeda Pharmaceuticals International, Inc. authors (BJP and OD) contributed primarily to refinement of study design, interpretation of data, and editing and revising of the initial drafts. All authors read and approved the final manuscript.

\section{Competing interests}

EK has consultant/advisor/grant recipient relationships with Takeda Pharmaceuticals International, Inc. (Deerfield, IL, USA). He has been a shareholder of Savient Pharmaceuticals, Inc. (East Brunswick, NJ, USA) and currently holds common stock in that company. He is an investigator for a clinical trial performed by Ardea Biosciences (San Diego, CA, USA). He serves on advisory boards for Takeda Pharmaceuticals International, Inc., URL Pharma (Philadelphia, PA, USA), and UCB (Brussels, Belgium). BJP and OD are employees of Takeda Pharmaceuticals International, Inc. LC declares that she has no competing interests.

Received: 2 December 2010 Revised: 23 February 2011

Accepted: 18 April 2011 Published: 18 April 2011

\section{References}

1. Baker JF, Krishnan E, Chen L, Schumacher HR: Serum uric acid and cardiovascular disease: recent developments, and where do they leave us? Am J Med 2005, 118:816-826.

2. Kanbay M, Sánchez-Losada LG, Franco M, Madero M, Solak Y, RodriguezIturbe B, Covoc A, Johnson RJ: Microvascular disease and its role in the brain and cardiovascular system: a potential role for uric acid as a cardiorenal toxin. Nephrol Dial Transplant 2011, 26:430-437.

3. Simons DB, Schwartz RS, Edwards WD, Sheedy PF, Breen JF, Rumberger JA: Noninvasive definition of anatomic coronary artery disease by ultrafast computed tomographic scanning: a quantitative pathologic comparison study. J Am Coll Cardiol 1992, 20:1118-1126.

4. Agatston AS, Janowitz WR, Hildner FJ, Zusmer NR, Viamonte M Jr, Detrano R: Quantification of coronary artery calcium using ultrafast computed tomography. J Am Coll Cardiol 1990, 15:827-832.

5. Budoff MJ, Georgiou D, Brody A, Agatston AS, Kennedy J, Wolfkiel C, Stanford W, Shields P, Lewis RJ, Janowitz WR, Rich S, Brundage BH: Ultrafast computed tomography as a diagnostic modality in the detection of coronary artery disease: a multicenter study. Circulation 1996, 93:898-904.

6. Folsom AR, Kronmal RA, Detrano RC, O'Leary DH, Bild DE, Bluemke DA, Budoff MJ, Liu K, Shea S, Szklo M, Tracy RP, Watson KE, Burke GL: Coronary artery calcification compared with carotid intima-media thickness in the 
prediction of cardiovascular disease incidence: the Multi-Ethnic Study of Atherosclerosis (MESA). Arch Intern Med 2008, 168:1333-1339.

7. Hughes $G H$, Cutter $G$, Donahue R, Friedman GD, Hulley $S$, Hunkeler $E$, Jacobs DR Jr, Liu K, Orden S, Pirie P, Tucker B, Wagenknecht L: Recruitment in the Coronary Artery Disease Risk Development in Young Adults (Cardia) Study. Control Clin Trials 1987, 8(4 Suppl):68S-73S.

8. Raggi P, Cooil B, Ratti C, Callister TQ, Budoff M: Progression of coronary artery calcium and occurrence of myocardial infarction in patients with and without diabetes mellitus. Hypertension 2005, 46:238-243.

9. American Diabetes Association: Diagnosis and classification of diabetes mellitus. Diabetes Care 2009, 32(Suppl 1):S62-67.

10. Levey AS, Bosch JP, Lewis JB, Greene T, Rogers N, Roth D: A more accurate method to estimate glomerular filtration rate from serum creatinine: a new prediction equation. Modification of Diet in Renal Disease Study Group. Ann Intern Med 1999, 130:461-470.

11. Carr JJ, Nelson JC, Wong ND, McNitt-Gray M, Arad Y, Jacobs DR Jr, Sidney S, Bild DE, Williams OD, Detrano RC: Calcified coronary artery plaque measurement with cardiac $\mathrm{CT}$ in population-based studies: standardized protocol of Multi-Ethnic Study of Atherosclerosis (MESA) and Coronary Artery Risk Development in Young Adults (CARDIA) study. Radiology 2005, 234:35-43.

12. Budoff MJ, Achenbach S, Blumenthal RS, Carr JJ, Goldin JG, Greenland P, Guerci AD, Lima JA, Rader DJ, Rubin GD, Shaw L, Wiegers SE, American Heart Association Committee on Cardiovascular Imaging and Intervention; American Heart Association Council on Cardiovascular Radiology and Intervention; American Heart Association Committee on Cardiac Imaging, Council on Clinical Cardiology: Assessment of coronary artery disease by cardiac computed tomography: a scientific statement from the American Heart Association Committee on Cardiovascular Imaging and Intervention, Council on Cardiovascular Radiology and Intervention, and Committee on Cardiac Imaging, Council on Clinical Cardiology. Circulation 2006, 114:1761-1791.

13. Tanaka M, Tomiyasu K, Fukui M, Akamabe $\mathrm{S}$, Kobayashi-Takenaka $\mathrm{Y}$, Nakano K, Kadono M, Hasegawa G, Oda Y, Nakamura N: Evaluation of characteristics and degree of remodeling in coronary atherosclerotic lesions by 64-detector multislice computed tomography (MSCT). Atherosclerosis 2009, 203:436-441.

14. Loria CM, Liu K, Lewis CE, Hulley SB, Sidney S, Schreiner PJ, Williams OD, Bild DE, Detrano R: Early adult risk factor levels and subsequent coronary artery calcification: the CARDIA Study. J Am Coll Cardiol 2007, 49:2013-2020.

15. Chobanian AV, Bakris GL, Black HR, Cushman WC, Green LA, Izzo JL Jr, Jones DW, Materson BJ, Oparil S, Wright JT Jr, Roccella EJ, Joint National Committee on Prevention, Detection, Evaluation, and Treatment of High Blood Pressure. National Heart, Lung, and Blood Institute; National High Blood Pressure Education Program Coordinating Committee: Seventh report of the Joint National Committee on Prevention, Detection, Evaluation, and Treatment of High Blood Pressure. Hypertension 2003, 42:1206-1252.

16. National Cholesterol Education Program (NCEP) Expert Panel on Detection, Evaluation, and Treatment of High Blood Cholesterol in Adults (Adult Treatment Panel III): Third Report of the National Cholesterol Education Program (NCEP) Expert Panel on Detection, Evaluation, and Treatment of High Blood Cholesterol in Adults (Adult Treatment Panel III) final report. Circulation 2002, 106:3143-3421.

17. Hosmer DW, Lemeshow S: Assessing the fit of the model. Applied Logistic Regression. 2 edition. New York: John Wiley \& Sons, Inc; 2000, 143-202.

18. Ames BN, Cathcart R, Schwiers E, Hochstein P: Uric acid provides an antioxidant defense in humans against oxidant- and radical-caused aging and cancer: a hypothesis. Proc Natl Acad Sci USA 1981, 78:6858-6862

19. Gagliardi AC, Miname MH, Santos RD: Uric acid: a marker of increased cardiovascular risk. Atherosclerosis 2009, 202:11-17.

20. Yildiz A, Yilmaz R, Demirbag R, Gur M, Bas MM, Erel O: Association of serum uric acid level and coronary blood flow. Coron Artery Dis 2007, 18:607-613.

21. Nieto FJ, Iribarren C, Gross MD, Comstock GW, Cutler RG: Uric acid and serum antioxidant capacity: a reaction to atherosclerosis? Atherosclerosis 2000, 148:131-139

22. Rodrigues TC, Maahs DM, Johnson RJ, Jalal DI, Kinney GL, Rivard C, Rewers M, Snell-Bergeon JK: Serum uric acid predicts progression of subclinical coronary atherosclerosis in individuals without renal disease. Diabetes Care 2010, 33:2471-2473.

23. Shemesh J, Morag-Koren N, Goldbourt U, Grossman E, Tenenbaum A, Fisman EZ, Apter S, Itzchak Y, Motro M: Coronary calcium by spiral computed tomography predicts cardiovascular events in high-risk hypertensive patients. J Hypertens 2004, 22:605-610.

24. Pacifico L, Cantisani V, Anania C, Bonaiuto E, Martino F, Pascone R, Chiesa C: Serum uric acid and its association with metabolic syndrome and carotid atherosclerosis in obese children. Eur J Endocrinol 2009, 160:45-52.

25. Coutinho T de A, Turner ST, Peyser PA, Bielak LF, Sheedy PF, Kullo IJ: Associations of serum uric acid with markers of inflammation, metabolic syndrome, and subclinical coronary atherosclerosis. Am J Hypertens 2007, 20:83-89.

26. Mitsutake R, Miura S, Shiga Y, Kawamura A, Saku K: Is chronic kidney disease associated with coronary artery stenosis or calcification as assessed by multi-detector row computed tomography? Intern Med 2008, 47:1835-1841.

27. Altunkan S, Erdogan N, Altin L, Budoff MJ: Relation of coronary artery calcium to left ventricular mass and geometry in patients with essential hypertension. Blood Press Monit 2003, 8:9-15.

28. Neogi T, Terkeltaub R, Ellison RC, Hunt S, Zhang Y: Serum urate is not associated with coronary artery calcification: The NHLBI Family Heart Study. J Rheumatol 2011, 38:111-117.

29. Tanaka M, Fukui M, Tomiyasu Kl, Akabame S, Nakano K, Hasegawa G, Oda Y, Nakamura N: Low serum bilirubin concentration is associated with coronary artery calcification (CAC). Atherosclerosis 2009, 206:287-291.

30. Devries S, Wolfkiel C, Fusman B, Bakdash H, Ahmed A, Levy P, Chomka E, Kondos $G$, Zajac E, Rich S: Influence of age and gender on the presence of coronary calcium detected by ultrafast computed tomography. J Am Coll Cardiol 1995, 25:76-82

31. Frink RJ: Gender gap, inflammation and acute coronary disease: are women resistant to atheroma growth? Observations at autopsy. Invasive Cardiol 2009, 21:270-277.

32. Iribarren C, Folsom AR, Eckfeldt JH, McGovern PG, Nieto FJ: Correlates of uric acid and its association with asymptomatic carotid atherosclerosis: the ARIC Study. Atherosclerosis Risk in Communities. Ann Epidemiol 1996, 6:331-340.

33. Ishizaka N, Ishizaka Y, Toda E, Nagai R, Yamakado M: Association between serum uric acid, metabolic syndrome, and carotid atherosclerosis in Japanese individuals. Arterioscler Thromb Vasc Biol 2005, 25:1038-1044.

34. Krishnan E, Baker JF, Furst DE, Schumacher HR: Gout and the risk of acute myocardial infarction. Arthritis Rheum 2006, 54:2688-2696.

35. Jalal DI, Rivard CJ, Johnson RJ, Maahs DM, McFann K, Rewers M, SnellBergeon JK: Serum uric acid levels predict the development of albuminuria over 6 years in patients with type 1 diabetes: findings from the Coronary Artery Calcification in Type 1 Diabetes study. Nephrol Dial Transplant 2010, 25:1865-1869.

\section{doi:10.1186/ar3322}

Cite this article as: Krishnan et al: Hyperuricemia and the risk for subclinical coronary atherosclerosis - data from a prospective observational cohort study. Arthritis Research \& Therapy 2011 13:R66.

\section{Submit your next manuscript to BioMed Central and take full advantage of:}

- Convenient online submission

- Thorough peer review

- No space constraints or color figure charges

- Immediate publication on acceptance

- Inclusion in PubMed, CAS, Scopus and Google Scholar

- Research which is freely available for redistribution 